\title{
A NEW WEAK GALERKIN FINITE ELEMENT METHOD FOR ELLIPTIC INTERFACE PROBLEMS
}

\author{
LIN MU*, JUNPING WANG ${ }^{\dagger}$, XIU YE YE $^{\ddagger}$ AND SHAN ZHAO
}

\begin{abstract}
A new weak Galerkin (WG) finite element method is introduced and analyzed in this paper for solving second order elliptic equations with discontinuous coefficients and interfaces. Comparing with the existing WG algorithm for solving the same type problems, the present WG method has a simpler variational formulation and fewer unknowns. Moreover, the new WG algorithm allows the use of finite element partitions consisting of general polytopal meshes and can be easily generalized to high orders. Optimal order error estimates in both $H^{1}$ and $L^{2}$ norms are established for the present WG finite element solutions. Extensive numerical experiments have been conducted to examine the accuracy, flexibility, and robustness of the proposed WG interface approach. In solving regular elliptic interface problems, high order of convergences are numerically confirmed by using piecewise polynomial basis functions of high degrees. Moreover, the WG method is shown to be able to accommodate very complicated interfaces, due to its flexibility in choosing finite element partitions. Finally, in dealing with challenging problems with low regularities, the piecewise linear WG method is capable of delivering a second order of accuracy in $L_{\infty}$ norm for both $C^{1}$ and $H^{2}$ continuous solutions.
\end{abstract}

Key words. Finite element method, weak Galerkin method, elliptic interface problem, nonsmooth interface, low solution regularity, high order method.

AMS subject classifications. Primary, 65N15, 65N30, 76D07; Secondary, 35B45, 35J50

1. Introduction. In this paper, we will develop a novel weak Galerkin (WG) finite element method for solving a typical two-dimensional (2D) elliptic interface problem

$$
\begin{aligned}
& -\nabla \cdot A \nabla u=f, \quad \text { in } \Omega, \\
& u=g, \quad \text { on } \partial \Omega \backslash \Gamma, \\
& \llbracket u \rrbracket_{\Gamma}=\left.u\right|_{\Omega_{1}}-\left.u\right|_{\Omega_{2}}=\psi, \quad \text { on } \Gamma, \\
& \llbracket A \nabla u \cdot \mathbf{n} \rrbracket_{\Gamma}=\left.A_{1} \nabla u\right|_{\Omega_{1}} \cdot \mathbf{n}_{1}+\left.A_{2} \nabla u\right|_{\Omega_{2}} \cdot \mathbf{n}_{2}=\phi, \quad \text { on } \Gamma \text {, }
\end{aligned}
$$

where $\Omega=\Omega_{1} \cup \Omega_{2}, \Gamma=\Omega_{1} \cap \Omega_{2}$, and $\mathbf{n}_{1}$ and $\mathbf{n}_{2}$ denote unit outward normals of $\Omega_{1}$ and $\Omega_{2}$. Across the interface $\Gamma$ which could be of Lipschitz continuous, the elliptic coefficient $A$ is discontinuous and the source $f$ can be singular. Based on a much simpler formulation, the proposed WG finite element method will significantly

* Computer Science and Mathematics Division, Oak Ridge National Laboratory, Oak Ridge, TN, 37831 (mul1@ornl.gov). The first author's research is based upon work supported in part by the U.S. Department of Energy, Office of Science, Office of Advanced Scientific Computing Research, Applied Mathematics program under award number ERKJE45; and by the Laboratory Directed Research and Development program at the Oak Ridge National Laboratory, which is operated by UT-Battelle, LLC., for the U.S. Department of Energy under Contract DE-AC05-00OR22725.

${ }^{\dagger}$ Division of Mathematical Sciences, National Science Foundation, Arlington, VA 22230 (jwang@ nsf.gov). The research of Wang was supported by the NSF IR/D program, while working at the Foundation. However, any opinion, finding, and conclusions or recommendations expressed in this material are those of the author and do not necessarily reflect the views of the National Science Foundation.

${ }^{\ddagger}$ Department of Mathematics and Statistics, University of Arkansas at Little Rock, Little Rock, AR 72204 (xxye@ualr.edu). This research of Ye was supported in part by National Science Foundation Grant DMS-1115097.

$\S$ Department of Mathematics, University of Alabama, Tuscaloosa, AL 35487 (szhao@ua.edu). This research of Zhao was supported in part by National Science Foundation Grant DMS-1318898 and the University of Alabama Research Stimulation Program (RSP) award.

1

(C) 2016. This manuscript version is made available under the Elsevier user license http://www.elsevier.com/open-access/userlicense/1.0/ 
outperform our previous WG algorithm for solving elliptic interface problems [37], in terms of both robustness and accuracy.

Elliptic interface problems with discontinuous coefficients and singular sources are found in many applications, such as fluid mechanics [31], computational electromagnetics [18, 23, 56, 55], materials science [25, 29], and biological science [54, 15, 8]. Since the physical solutions to elliptic interface problems are usually non-smooth or even discontinuous across the interface, the standard numerical methods ofter perform poorly. Generally speaking, to secure the accuracy near the interface, the jump conditions (1.3) and (1.4) have to be incorporated into the numerical discretization in certain manner.

The finite element solution of elliptic interface problems dates back to 1970s [1], and has attracted much research interest in the past few decades [4, 10, 13, 42]. In classical finite element methods (FEMs), the irregular geometry is naturally handled by using unstructured meshes that conform to the interface $\Gamma$ and boundary $\partial \Omega$. The performance of such kind of interface-fitted FEMs depends not only on the quality of underlying finite element partition, but also on the variational formulation of the problem. Because the interface jump conditions are usually not satisfied in common FEM solution spaces, linear constraints based on either penalty methods or Lagrange multipliers have been constructed $[16,6]$ to impose these conditions in variational formulations. Similarly, Galerkin projections and numerical fluxes have been considered in discontinuous Galerkin (DG) variational formulations [12, 17, 21, 30] to weakly enforce the interface conditions. Recently, by approximating differential operators by their weak forms as distributions and treating jump conditions appropriately, a weak Galerkin (WG) FEM has been developed in [37] for solving elliptic interface problems. For moving interfaces and some exotically complex topologies, the construction of high quality FEM meshes could be difficult and time-consuming. This motivates the development of various embedded or immersed FEMs [13, 19, 26, 47, 20, 27, 35, 28, 49, 50], in which the interface is allowed to cut through finite elements. In immersed FEMs, structured Cartesian meshes can be simply employed so that the time-consuming mesh generation process is avoided, while a sophisticated interface algorithm is indispensable to accommodate the complex interface geometries and jump conditions. In original immersed FEMs [13], this is achieved through modifying the basis functions inside cut-through elements to weakly satisfy jump conditions. Other interface approaches, such as enforcing jump conditions via fictitious nodes [49, 50] and Lagrange multipliers $[26,20,27,28]$ have also been developed. Convergence analysis of the regular and immersed FEMs for solving elliptic interface problems has been considered by many authors $[12,24,44,7]$.

The development of finite difference methods for solving elliptic interface problems based on Cartesian grids has also received much attention in the past a few decades. Peskin pioneered the field by introducing a first order accurate immersed boundary method in 1977 [41, 40]. The second order Cartesian grid method for solving elliptic interface problems was first presented by LeVeque and $\mathrm{Li}$ in 1994 [32, 34]. In their immersed interface method (IIM), the jump conditions are appropriately imposed via local Taylor's series expansions. In recent years, many other elegant finite difference interface methods have been developed, such as the ghost fluid method proposed by Fedkiw, Osher and coworkers [14], maximum principle preserving and explicit jump IIMs [33, 48, 3, 34], coupling interface method [11], piecewise-polynomial interface method [9], and matched interface and boundary (MIB) method [56, 57, 52]. Besides finite element and finite difference methods, other effective algorithms for solving 
elliptic interface problems include integral equation methods [36, 51], finite volume method [39], and virtual node method [2, 22].

Even though a great success has been achieved in the aforementioned studies for solving elliptic interface problems, there are still several hot research topics in the field that call for further development. In particular, challenges remained in designing algorithms for treating very complicated interface geometries, for problems with low regularity solutions, and to achieve higher order of convergence [37]. Recent advances in these directions are briefly reviewed below.

The material interfaces in real applications can be geometrically complicated and very irregular. In some extreme cases with nonsmooth interfaces or interfaces with Lipschitz continuity, geometric singularities, such as sharp edges, cusps and tips, could be encountered. For the interface-fitted FEMs, in order to deal with complex interfaces, it is desired that the variational formulation does not critically depend on the quality of underlying finite element partitions. DG [30] and WG [37] FEMs that potentially allow poorly-generated meshes and/or hanging nodes are thus promising in resolving geometrically complicated interfaces. For the unfitted Cartesian grid methods, significant advance has been made in the literature in the past decade on treating arbitrary interfaces with Lipschitz continuity. The first second order accurate finite difference scheme that is capable to deal with arbitrary nonsmooth interfaces was developed within the matched interface and boundary (MIB) framework in 2007 [53]. The 2D MIB interface techniques have been generalized to 3D in [54], giving rise to a second order accurate 3D MIB scheme for arbitrary complex interfaces. The 3D MIB scheme finds great success in protein electrostatic analysis [54, 15, 8] and has been recently formulated in the Galerkin formulation $[49,50]$. Other second order accurate approaches for arbitrarily complex geometry include the piecewisepolynomial interface method in 2D [9], the immersed FEM in 2D [26, 27] and 3D [28], and the embedded method in 2D [2] and 3D [22]. The development of Cartesian grid schemes with orders greater than two for arbitrarily shaped nonsmooth interfaces or geometric singularities remains to be an open problem [37].

In real applications, the geometric singularities of interfaces are usually associated with the loss of regularities in physical solutions [37]. This motivates some studies on solving elliptic interface problems with low solution regularities. For instance, Hou and his coworkers have examined the convergence of their immersed FEMs based on non-body-fitted grids for solving Poisson equations with the interface being $C^{1}$ or Lipschitz continuous [27]. The orders of convergence in $L_{\infty}$ norm of their FEM are found to be 1.5 and 0.65 , respectively, in the solution and the gradient of the solution, when the solution is $H^{2}$ continuous, while such orders become 1 and 0.75 , respectively, when the solution is just $C^{1}$ continuous. Such orders are the best known results for the immersed FEMs, in which the triangles are allowed to cut through the interfaces [27]. With an additional computational cost for generating body-fitted grids, the WG FEM has been applied to solve similar problems with $C^{1}$ or Lipschitz continuous interfaces, and achieves a uniform first order of convergence in $L_{\infty}$ norm for the gradient of the solution, no matter the solution is $C^{1}$ or $H^{2}$ continuous [37]. For the solution itself, the WG method attains a second order and 1.75th order in $L_{\infty}$ norm for solutions being $H^{2}$ and $C^{1}$ continuous, respectively. Such orders are some of the best results for elliptic interface problems with low solution regularities induced by geometric singularities.

In developing numerical methods for elliptic interface problems, higher order of convergence is always one of the major research goals, because high order methods 
are more accurate and cost-efficient. For Cartesian grid methods, in which the interface could cut the uniform grid in an arbitrary manner, the first second order accurate method developed in the literature is the immersed interface method (IIM) due to LeVeque and Li [32]. Numerous second order Cartesian grid methods have been developed since then, but only a few schemes have potentials to converge in a faster rate in solving elliptic interface problems. The four order formulation of the IIM can be constructed, provided that high order jump conditions are available [34]. Through iteratively using zero and first order jump conditions, a matched interface and boundary (MIB) method has been developed in [57], which normally achieves a fourth order of convergence in resolving curved interfaces based on simple Cartesian grids and could reach up to sixth order when the interfaces are smooth enough. The high order MIB schemes have been successfully generalized to 3D [52], as well as for solving electromagnetic interface problems governed by the Helmholtz equation [55]. To achieve a higher order of convergence in the body-fitted FEMs, in which unstructured meshes conform to the interfaces, a proper variational formulation that handles jumps in solution and flux is indispensable. By using piecewise linear basic functions, the previous WG method [37] attains a third order of convergence in both $L^{2}$ and $L_{\infty}$ norms for a simple circular interface problem. By using superparametric elements at the interface, the hybridizable DG method [30] is able to deliver the optimal order of $k+1$ in the $L^{2}$ norm for several polynomial orders of $k$.

The goal of this work is to develop a new WG algorithm which significantly advances our previous WG method [37] in formulations and partitions for solving elliptic interface problems. The WG-FEM refers to a general finite element technique for partial differential equations where differential operators (e.g., gradient, divergence, curl, Laplacian) are approximated by their weak forms as distributions. The first WG-FEM was introduced by Wang and Ye [45] for solving second order elliptic equations based on a discrete weak gradient arising from local RT [43] or BDM [5] elements. By using a Lagrange multiplier to handle the interface conditions, the WG algorithm has been successfully applied in [37] for solving elliptic interface problems. However, the WG interface algorithm [37] is based on the original WG method [45] and is limited to classical finite element partitions of triangles in 2D and tetrahedra in 3D. Recently, a mixed WG-FEM has been developed in [46] for the second order elliptic equation, in which the use of stabilization for the flux variable plays a key role in the mixed formulation. The resulting WG mixed finite element schemes turned out to be applicable for general partitions consisting of shape regular polytopes, such as polygons in 2D and polyhedra in 3D. More recently, a new WG-FEM [38] which discretizes the second order elliptic equation in non-mixed form directly, has been constructed to admit general finite element partitions consisting of arbitrary polytopal elements.

A weak Galerkin method was introduced in [37] for the elliptic interface problem using a Lagrange multiplier to handle the interface conditions that leads to a saddle point system. In this paper, a new weak Galerkin method using a parameter free stabilizer has been developed for the same problem. Compared with the WG method in [37], this method has many new features including symmetric positive definite formulation, fewer unknowns and, more importantly, allowing the use of general meshes such as hybrid meshes, polygonal and polyhedral meshes and meshes with hanging nodes. These features make the new WG-FEM more flexible in handling complicated interface geometries.

The rest of this paper is organized as follows. A new weak Galerkin formulation for the elliptic interface problem (1.1)-(1.4) will be proposed in Section 2. A conver- 
gence analysis of the new WG method will be presented in Section 3 . In Section 4, we examine the numerical performance of the proposed WG-FEM for solving elliptic interface problems. Besides test problems that admit solutions with low regularities, we will consider a few examples with very complicated interfaces. High order convergence will also be illustrated. Finally, this paper ends with a conclusion.

2. Methods and Algorithms. Let $\mathcal{T}_{h}$ be a partition of the domain $\Omega$ consisting of polygons in two dimension satisfying a set of conditions specified in [46]. Denote by $\mathcal{E}_{h}$ the set of all edges in $\mathcal{T}_{h}$, and let $\mathcal{E}_{h}^{0}=\mathcal{E}_{h} \backslash \partial \Omega$ be the set of all interior edges. Let $\Gamma_{h}$ be the subset of $\mathcal{E}_{h}$ of all edges on $\Gamma$. For every element $T \in \mathcal{T}_{h}$, we denote by $h_{T}$ its diameter and mesh size $h=\max _{T \in \mathcal{T}_{h}} h_{T}$ for $\mathcal{T}_{h}$.

The weak Galerkin methods create a new way to define a function $v$ that allows $v$ taking different forms in the interior and on the boundary of the element:

$$
v= \begin{cases}v_{0}, & \text { in } T^{0} \\ v_{b}, & \text { on } \partial T\end{cases}
$$

where $T_{0}$ denotes the interior of $T$. Since our weak function $v$ is formed by two parts $v_{0}$ and $v_{b}$, we write $v$ as $v=\left\{v_{0}, v_{b}\right\}$ in short without confusion.

For a given integer $k \geq 1$, let $V_{h}$ be the WG finite element space associated with $\mathcal{T}_{h}$ defined as follows

$$
V_{h}=\left\{v=\left\{v_{0}, v_{b}\right\}:\left.v_{0}\right|_{T} \in P_{k}(T),\left.v_{b}\right|_{e} \in P_{k}(e), e \in \partial T, T \in \mathcal{T}_{h}\right\}
$$

and

$$
V_{h}^{0}=\left\{v: v \in V_{h}, v_{b}=0 \text { on } \partial \Omega\right\} .
$$

We would like to emphasize that any function $v \in V_{h}$ has a single value $v_{b}$ on each edge $e \in \mathcal{E}_{h}$.

For each $v \in V_{h}$, the weak gradient of it, denoted by $\nabla_{w} v$, is defined as the unique polynomial $\left(\nabla_{w} v\right) \in\left[P_{k-1}(T)\right]^{2}$ satisfying the following equation

$$
\left(\nabla_{w} v, q\right)_{T}=-\left(v_{0}, \nabla \cdot q\right)_{T}+\left\langle v_{b}, q \cdot \mathbf{n}\right\rangle_{\partial T}, \quad \forall q \in\left[P_{k-1}(T)\right]^{2} .
$$

For each element $T \in \mathcal{T}_{h}$, denote by $Q_{0}$ the $L^{2}$ projection from $L^{2}(T)$ to $P_{k}(T)$ and by $Q_{b}$ the $L^{2}$ projection from $L^{2}(e)$ to $P_{k}(e)$ for any $e \in \mathcal{E}_{h}$.

Now we introduce two forms on $V_{h}$ as follows:

$$
\begin{aligned}
& s(v, w)=\sum_{T \in \mathcal{T}_{h}} h^{-1}\left\langle v_{0}-v_{b}, w_{0}-w_{b}\right\rangle_{\partial T}, \\
& a(v, w)=\sum_{T \in \mathcal{T}_{h}}\left(A \nabla_{w} v, \nabla_{w} w\right)_{T}+s(v, w) .
\end{aligned}
$$

Let $e \subset \Gamma$ be shared by two elements $T_{1} \subset \Omega_{1}$ and $T_{2} \subset \Omega_{2}$. We defined three forms

$$
\begin{aligned}
\left\langle\psi, A \nabla_{w} v \cdot \mathbf{n}\right\rangle_{\Gamma} & =\sum_{e \in \Gamma_{h}}\left\langle\psi, A \nabla_{w}\left(\left.v\right|_{T_{2}}\right) \cdot \mathbf{n}\right\rangle_{e} \\
h^{-1}\left\langle\psi, v_{0}-v_{b}\right\rangle_{\Gamma} & =\sum_{e \in \Gamma_{h}} h^{-1}\left\langle\psi,\left.v_{0}\right|_{T_{2}}-v_{b}\right\rangle_{e} \\
\left\langle\phi, v_{b}\right\rangle_{\Gamma} & =\sum_{e \in \Gamma_{h}}\left\langle\phi, v_{b}\right\rangle_{e} .
\end{aligned}
$$


Weak Galerkin Algorithm 1. A numerical approximation for (1.1) and (1.4) can be obtained by seeking $u_{h}=\left\{u_{0}, u_{b}\right\} \in V_{h}$ satisfying both $u_{b}=Q_{b} g$ on $\partial \Omega$ and the following equation: for any $v=\left\{v_{0}, v_{b}\right\} \in V_{h}^{0}$

$$
a\left(u_{h}, v\right)=\left(f, v_{0}\right)+\left\langle\psi, A \nabla_{w} v \cdot \mathbf{n}\right\rangle_{\Gamma}+\left\langle\phi, v_{b}\right\rangle_{\Gamma}-h^{-1}\left\langle\psi, v_{0}-v_{b}\right\rangle_{\Gamma} .
$$

This WG method is symmetric, positive definite and parameter independent. It also conserves the mass.

3. Convergence Theory. The goal of this section is to provide a convergence theory for the weak Galerkin finite element method (WG-FEM) as described in the previous section. First, we will derive the equation that the error satisfies.

3.1. Error Equation. For simplicity of analysis, we assume that the coefficient $A$ in (1.1) is a piecewise constant with respect to the finite element partition $\mathcal{T}_{h}$.

Let $u_{h}=\left\{u_{0}, u_{b}\right\} \in V_{h}$ be the WG-FEM solution arising from the numerical scheme (2.6). Assume that the exact solution of (1.1)-(1.4) is given by $u$.

For an $e \in \mathcal{E}_{h}$ shared by the two elements $T_{1}$ and $T_{2}$, if $e \nsubseteq \Gamma,\left.u\right|_{T_{1} \cap e}=\left.u\right|_{T_{2} \cap e}$ and if $e \subset \Gamma,\left.u\right|_{T_{1} \cap e} \neq\left. u\right|_{T_{2} \cap e}$. Next we like to define $Q_{h} u=\left\{Q_{0} u, Q_{b} u\right\} \in V_{h}$. To ensure $Q_{h} u \in V_{h}$, i.e. $Q_{b} u$ takes single value on any $e \in \mathcal{E}_{h}$, we define $Q_{b} u$ in the following way: for $e \nsubseteq \Gamma$, let $Q_{b}=Q_{b}\left(\left.u\right|_{T_{1} \cap e}\right)=Q_{b}\left(\left.u\right|_{T_{2} \cap e}\right)$ and for $e \subset \Gamma$, let $Q_{b} u=Q_{b}\left(\left.u\right|_{T_{1} \cap e}\right)$ with $T_{1} \subset \Omega_{1}$ and $T_{2} \subset \Omega_{2}$. Thus $Q_{b}\left(\left.u\right|_{T_{2} \cap e}\right)=Q_{b} u-Q_{b} \psi$ on $e$.

Denote by $\mathbb{Q}_{h}$ the $L^{2}$ projection onto the local discrete gradient space $\left[P_{k-1}(T)\right]^{2}$. Then we have the following lemma.

Lemma 3.1. Let $Q_{h}$ and $\mathbb{Q}_{h}$ be the $L^{2}$ projection operators as defined. Then, on each element $T \in \mathcal{T}_{h}$, we have the following properties: for any $\tau \in\left[P_{k-1}(T)\right]^{2}$,

$$
\begin{aligned}
& (3.1)\left(\nabla_{w}\left(Q_{h} u\right), \tau\right)_{T}=\left(\mathbb{Q}_{h}(\nabla u), \tau\right)_{T}, \quad T \nsubseteq \Omega_{2} \quad \text { or } \quad \partial T \cap \Gamma=\emptyset \\
& (3.2)\left(\nabla_{w}\left(Q_{h} u\right), \tau\right)_{T}=\left(\mathbb{Q}_{h}(\nabla u), \tau\right)_{T}+\langle\psi, \tau \cdot \mathbf{n}\rangle_{\partial T \cap \Gamma}, T \subset \Omega_{2} \quad \text { and } \partial T \cap \Gamma \neq \emptyset .
\end{aligned}
$$

Proof. Using (2.3), the integration by parts and the definitions of $Q_{h}$ and $\mathbb{Q}_{h}$, we have that for any $\tau \in\left[P_{k-1}(T)\right]^{2}$ and for any $T \nsubseteq \Omega_{2}$ or $\partial T \cap \Gamma=\emptyset$,

$$
\begin{aligned}
\left(\nabla_{w}\left(Q_{h} u\right), \tau\right)_{T} & =-\left(Q_{0} u, \nabla \cdot \tau\right)_{T}+\left\langle Q_{b} u, \tau \cdot \mathbf{n}\right\rangle_{\partial T} \\
& =-(u, \nabla \cdot \tau)_{T}+\langle u, \tau \cdot \mathbf{n}\rangle_{\partial T} \\
& =(\nabla u, \tau)_{T} \\
& =\left(\mathbb{Q}_{h}(\nabla u), \tau\right)_{T},
\end{aligned}
$$

which implies the desired identity (3.1). For any $T \subset \Omega_{2}$ and $\partial T \cap \Gamma \neq \emptyset$, we have

$$
\begin{aligned}
\left(\nabla_{w}\left(Q_{h} u\right), \tau\right)_{T} & =-\left(Q_{0} u, \nabla \cdot \tau\right)_{T}+\left\langle Q_{b} u, \tau \cdot \mathbf{n}\right\rangle_{\partial T} \\
& =-(u, \nabla \cdot \tau)_{T}+\langle u, \tau \cdot \mathbf{n}\rangle_{\partial T}+\langle\psi, \tau \cdot \mathbf{n}\rangle_{\partial T \cap \Gamma} \\
& =(\nabla u, \tau)_{T}+\langle\psi, \tau \cdot \mathbf{n}\rangle_{\partial T \cap \Gamma} \\
& =\left(\mathbb{Q}_{h}(\nabla u), \tau\right)_{T}+\langle\psi, \tau \cdot \mathbf{n}\rangle_{\partial T \cap \Gamma} .
\end{aligned}
$$

We have proved the lemma.

Let

$$
e_{h}=\left\{e_{0}, e_{b}\right\}=\left\{Q_{0} u-u_{0}, Q_{b} u-u_{b}\right\}
$$


be the error between the WG finite element solution and the $L^{2}$ projection of the exact solution.

Define $\ell_{1}(u, v)$ and $\ell_{2}(u, v)$ as

$$
\begin{aligned}
& \ell_{1}(u, v)=\sum_{T \in \mathcal{T}_{h}}\left\langle A\left(\nabla u-\mathbb{Q}_{h} \nabla u\right) \cdot \mathbf{n}, v_{0}-v_{b}\right\rangle_{\partial T}, \\
& \ell_{2}(u, v)=\sum_{T \in \mathcal{T}_{h}} h^{-1}\left\langle Q_{0} u-Q_{b}\left(\left.u\right|_{T}\right), v_{0}-v_{b}\right\rangle_{\partial T} .
\end{aligned}
$$

LEMmA 3.2. Let $e_{h}$ be the error of the weak Galerkin finite element solution arising from (2.6). Then, for any $v \in V_{h}^{0}$ we have

$$
a\left(e_{h}, v\right)=-\ell_{1}(u, v)+\ell_{2}(u, v) .
$$

Proof. It follows from the integration by parts, (3.1) and (3.2),

$$
\begin{aligned}
-\left(\nabla \cdot A \nabla u, v_{0}\right) & =\sum_{T \in \mathcal{T}_{h}}(A \nabla u, \nabla v)_{T}-\sum_{T \in \mathcal{T}_{h}}\left\langle A \nabla u \cdot \mathbf{n}, v_{0}\right\rangle_{\partial T} \\
& =\sum_{T \in \mathcal{T}_{h}}\left(A \nabla u, \nabla v_{0}\right)_{T}-\sum_{T \in \mathcal{T}_{h}}\left(\left\langle A \nabla u \cdot \mathbf{n}, v_{0}-v_{b}\right\rangle_{\partial T}-\left\langle A \nabla u \cdot \mathbf{n}, v_{b}\right\rangle_{\partial T}\right) \\
& =\sum_{T \in \mathcal{T}_{h}}\left(A \nabla u, \nabla v_{0}\right)_{T}-\sum_{T \in \mathcal{T}_{h}}\left\langle A \nabla u \cdot \mathbf{n}, v_{0}-v_{b}\right\rangle_{\partial T}-\left\langle\phi, v_{b}\right\rangle_{\Gamma} \\
& =\sum_{T \in \mathcal{T}_{h}}\left(A \mathbb{Q}_{h} \nabla u, \nabla_{w} v\right)_{T}-\sum_{T \in \mathcal{T}_{h}}\left\langle A \nabla u \cdot \mathbf{n}-A \mathbb{Q}_{h} \nabla u \cdot \mathbf{n}, v_{0}-v_{b}\right\rangle_{\partial T}-\left\langle\phi, v_{b}\right\rangle_{\Gamma} \\
& =\sum_{T \in \mathcal{T}_{h}}\left(A \nabla_{w} Q_{h} u, \nabla_{w} v\right)_{T}-\sum_{T \in \mathcal{T}_{h}}\left\langle A \nabla u \cdot \mathbf{n}-A \mathbb{Q}_{h} \nabla u \cdot \mathbf{n}, v_{0}-v_{b}\right\rangle_{\partial T} \\
& -\left\langle\phi, v_{b}\right\rangle_{\Gamma}-\left\langle\psi, A \nabla_{w} v \cdot \mathbf{n}\right\rangle_{\Gamma} \\
& =\sum_{T \in \mathcal{T}_{h}}\left(A \nabla_{w} Q_{h} u, \nabla_{w} v\right)_{T}+\ell_{1}(u, v)-\left\langle\psi, A \nabla_{w} v \cdot \mathbf{n}\right\rangle_{\Gamma}-\left\langle\phi, v_{b}\right\rangle_{\Gamma} . \\
(3.4) &
\end{aligned}
$$

Then we have

$$
\left(A \nabla_{w} Q_{h} u, \nabla_{w} v\right)=\left(f, v_{0}\right)-\ell_{1}(u, v)+\left\langle\psi, A \nabla_{w} v \cdot \mathbf{n}\right\rangle_{\Gamma}-\left\langle\phi, v_{b}\right\rangle_{\Gamma} .
$$

Adding $s\left(Q_{h} u, v\right)$ to both sides of the above equation gives

$$
a\left(Q_{h} u, v\right)=\left(f, v_{0}\right)-\ell_{1}(u, v)+s\left(Q_{h} u, v\right)+\left\langle\psi, A \nabla_{w} v \cdot \mathbf{n}\right\rangle_{\Gamma}+\left\langle\phi, v_{b}\right\rangle_{\Gamma} .
$$

For any $T \nsubseteq \Omega_{2}$ or $\partial T \cap \Gamma=\emptyset$, we have

$$
\left\langle Q_{0} u-Q_{b} u, v_{0}-v_{b}\right\rangle_{\partial T}=\left\langle Q_{0} u-Q_{b}\left(\left.u\right|_{T}\right), v_{0}-v_{b}\right\rangle_{\partial T},
$$

and for any $T \subset \Omega_{2}$ and $\partial T \cap \Gamma \neq \emptyset$, we have

$$
\left\langle Q_{0} u-Q_{b} u, v_{0}-v_{b}\right\rangle_{\partial T}=\left\langle Q_{0} u-Q_{b}\left(\left.u\right|_{T}\right), v_{0}-v_{b}\right\rangle_{\partial T}-\left\langle\psi, v_{0}-v_{b}\right\rangle_{\partial T \cap \Gamma} .
$$

Combining the two equations above, we obtain

$$
s\left(Q_{h} u, v\right)=\sum_{T \in \mathcal{T}_{h}} h^{-1}\left\langle Q_{0} u-Q_{b}\left(\left.u\right|_{T}\right), v_{0}-v_{b}\right\rangle_{\partial T}-h^{-1}\left\langle\psi, v_{0}-v_{b}\right\rangle_{\Gamma} .
$$


Substituting the above equation in (3.6) yields

$$
\begin{aligned}
a\left(Q_{h} u, v\right) & =\left(f, v_{0}\right)-\ell_{1}(u, v)+\ell_{2}(u, v)+\left\langle\psi, A \nabla_{w} v \cdot \mathbf{n}\right\rangle_{\Gamma} \\
& +\left\langle\phi, v_{b}\right\rangle_{\Gamma}-h^{-1}\left\langle\psi, v_{0}-v_{b}\right\rangle_{\Gamma} .
\end{aligned}
$$

The procedure of obtaining the equation above can be used to derive the WG formulation (2.6) by replacing $Q_{h} u$ by $u_{h}$ and dropping the two high order terms $\ell_{1}(u, v)$ and $\ell_{2}(u, v)$.

The difference of the above equation and (2.6) implies (3.3).

3.2. Error Estimates. For any $v \in V_{h}$, let

$$
\|v\|=\sqrt{a(v, v)}
$$

LEMma 3.3. The semi-norm $\|\cdot\|$ defined in (3.8) is a norm in $V_{h}^{0}$.

Proof. To prove the lemma, we need to show that $\|v\|=0$ implies $v=0$ for all $v \in V_{h}^{0}$. It follows from $\|v\|=0$,

$$
\left(A \nabla_{w} v, \nabla_{w} v\right)+\sum_{T \in \mathcal{T}_{h}} h_{T}^{-1}\left\langle v_{0}-v_{b}, v_{0}-v_{b}\right\rangle_{\partial T}=0
$$

which implies that $\nabla_{w} v=0$ on each element $T$ and $v_{0}=v_{b}$ on $\partial T$. It follows from $\nabla_{w} v=0$ and $(2.3)$ that for any $q \in\left[P_{k-1}(T)\right]^{2}$

$$
\begin{aligned}
0 & =\left(\nabla{ }_{w} v, q\right)_{T} \\
& =\left(\nabla v_{0}, q\right)_{T}-\left\langle v_{0}-v_{b}, q \cdot \mathbf{n}\right\rangle_{\partial T} \\
& =\left(\nabla v_{0}, q\right)_{T}-\left\langle Q_{b} v_{0}-v_{b}, q \cdot \mathbf{n}\right\rangle_{\partial T} \\
& =\left(\nabla v_{0}, q\right)_{T} .
\end{aligned}
$$

Letting $q=\nabla v_{0}$ in the equation above yields $\nabla v_{0}=0$ on $T \in \mathcal{T}_{h}$. Thus, $v_{0}=$ const on every $T \in \mathcal{T}_{h}$. This, together with the fact that $v_{0}=v_{b}$ on $\partial T$ and $v_{b}=0$ on $\partial \Omega$, implies that $v=0$. We have proved the lemma.

Let $T$ be an element with $e$ as an edge. For any function $\varphi \in H^{1}(T)$, the following trace inequality holds true (see [46] for details):

$$
\|\varphi\|_{e}^{2} \leq C\left(h_{T}^{-1}\|\varphi\|_{T}^{2}+h_{T}\|\nabla \varphi\|_{T}^{2}\right)
$$

LEMmA 3.4. Assume that $\mathcal{T}_{h}$ is shape regular. Then for $u \in H^{k+1}\left(\Omega_{i}\right)$ with $i=1,2$ and $v=\left\{v_{0}, v_{b}\right\} \in V_{h}$, we have

$$
\begin{aligned}
& \left|\ell_{1}(u, v)\right| \leq C h^{k}\left(\|u\|_{k+1, \Omega_{1}}+\|u\|_{k+1, \Omega_{2}}\right)\|v\| \\
& \left|\ell_{2}(u, v)\right| \leq C h^{k}\left(\|u\|_{k+1, \Omega_{1}}+\|u\|_{k+1, \Omega_{2}}\right)\|v\| .
\end{aligned}
$$

Proof. As to (3.10), it follows from the Cauchy-Schwarz inequality and the trace 
inequality (3.9) that

$$
\begin{aligned}
\left|\ell_{1}(u, v)\right| & =\left|\sum_{T \in \mathcal{T}_{h}}\left\langle A\left(\nabla u-\mathbb{Q}_{h} \nabla u\right) \cdot \mathbf{n}, v_{0}-v_{b}\right\rangle_{\partial T}\right| \\
& \leq C \sum_{T \in \mathcal{T}_{h}}\left\|A\left(\nabla u-\mathbb{Q}_{h} \nabla u\right)\right\|_{\partial T}\left\|v_{0}-v_{b}\right\|_{\partial T} \\
& \leq C\left(\sum_{T \in \mathcal{T}_{h}} h_{T}\left\|A\left(\nabla u-\mathbb{Q}_{h} \nabla u\right)\right\|_{\partial T}^{2}\right)^{\frac{1}{2}}\left(\sum_{T \in \mathcal{T}_{h}} h_{T}^{-1}\left\|v_{0}-v_{b}\right\|_{\partial T}^{2}\right)^{\frac{1}{2}} \\
& \leq C h^{k}\left(\|u\|_{k+1, \Omega_{1}}+\|u\|_{k+1, \Omega_{2}}\right)\|v\| .
\end{aligned}
$$

Using the definition of $Q_{b}$ and (3.9), we obtain

$$
\begin{aligned}
\left|\ell_{2}(u, v)\right| & =\left|\sum_{T \in \mathcal{T}_{h}} h_{T}^{-1}\left\langle Q_{0} u-Q_{b}\left(\left.u\right|_{T}\right), v_{0}-v_{b}\right\rangle_{\partial T}\right| \\
& =\left|\sum_{T \in \mathcal{T}_{h}} h_{T}^{-1}\left\langle Q_{0} u-u, v_{0}-v_{b}\right\rangle_{\partial T}\right| \\
& \leq C\left(\sum_{T \in \mathcal{T}_{h}}\left(h_{T}^{-2}\left\|Q_{0} u-u\right\|_{T}^{2}+\left\|\nabla\left(Q_{0} u-u\right)\right\|_{T}^{2}\right)\right)^{\frac{1}{2}} . \\
& \left(\sum_{T \in \mathcal{T}_{h}} h_{T}^{-1}\left\|v_{0}-v_{b}\right\|_{\partial T}^{2}\right)^{\frac{1}{2}} \\
\leq & C h^{k}\left(\|u\|_{k+1, \Omega_{1}}+\|u\|_{k+1, \Omega_{2}}\right)\|v\| .
\end{aligned}
$$

The proof is completed.

Now we are ready to derive the error bound for the WG-FEM approximation.

THEOREM 3.5. Let $u_{h} \in V_{h}$ be the weak Galerkin finite element solution of the problem (1.1)-(1.4) arising from (2.6). Assume the exact solution $u \in H^{k+1}\left(\Omega_{i}\right)$ with $i=1,2$. Then, there exists a constant $C$ such that

$$
\left\|u_{h}-Q_{h} u\right\| \leq C h^{k}\left(\|u\|_{k+1, \Omega_{1}}+\|u\|_{k+1, \Omega_{2}}\right) .
$$

Proof. By letting $v=e_{h}$ in (3.3), we have

$$
\left\|e_{h}\right\|^{2}=\ell_{1}\left(u, e_{h}\right)+\ell_{2}\left(u, e_{h}\right) .
$$

It then follows from (3.10) and (3.11) that

$$
\left\|e_{h}\right\|^{2} \leq C h^{k}\left(\|u\|_{k+1, \Omega_{1}}+\|u\|_{k+1, \Omega_{2}}\right)\left\|e_{h}\right\| \|
$$

which implies (3.13). This completes the proof.

4. Numerical experiments. In this section, we will explore the performance of the proposed weak Galerkin (WG) method in solving elliptic interface problems. Particular attentions will be paid on verifying its high order convergence and examining its robustness in dealing with low regularity solutions and complex geometries. The 
TABLE 4.1

Numerical convergence of the piecewise linear WG-FEM for Example 1.

\begin{tabular}{|c|c|cc|cc|}
\hline Mesh & \multirow{2}{*}{$\max \{h\}$} & \multicolumn{2}{|c|}{ Gradient } & \multicolumn{2}{c|}{ Solution } \\
\cline { 3 - 6 } & & $L^{2}$ error & order & $L^{2}$ error & order \\
\hline Level 1 & $5.6522 \mathrm{e}-1$ & $2.1027 \mathrm{e}-0$ & & $1.1644 \mathrm{e}-0$ & \\
Level 2 & $2.8553 \mathrm{e}-1$ & $6.8717 \mathrm{e}-1$ & 1.6378 & $3.2266 \mathrm{e}-1$ & 1.8794 \\
Level 3 & $1.5110 \mathrm{e}-1$ & $2.7346 \mathrm{e}-1$ & 1.4479 & $8.2758 \mathrm{e}-2$ & 2.1381 \\
Level 4 & $7.7543 \mathrm{e}-2$ & $1.2506 \mathrm{e}-1$ & 1.1728 & $2.0822 \mathrm{e}-2$ & 2.0685 \\
Level 5 & $3.9258 \mathrm{e}-2$ & $6.0641 \mathrm{e}-2$ & 1.0634 & $5.2136 \mathrm{e}-3$ & 2.0344 \\
\hline
\end{tabular}

TABLE 4.2

Numerical convergence of high order WG-FEMs for Example 1.

\begin{tabular}{|c|cc|cc|cc|}
\hline $\mathrm{k}$ & Mesh & $\max \{h\}$ & \multicolumn{2}{c|}{ Gradient } & \multicolumn{2}{c|}{ Solution } \\
\cline { 4 - 7 } & & & $L^{2}$ error & order & edge-based $L^{2}$ error & order \\
\hline 2 & Level 1 & $5.6522 \mathrm{e}-1$ & $1.7183 \mathrm{e}-1$ & & $6.6842 \mathrm{e}-2$ & \\
& Level 2 & $2.8553 \mathrm{e}-1$ & $4.1116 \mathrm{e}-2$ & 2.0943 & $6.8105 \mathrm{e}-3$ & 3.3445 \\
& Level 3 & $1.5110 \mathrm{e}-1$ & $1.0173 \mathrm{e}-2$ & 2.1946 & $7.6276 \mathrm{e}-4$ & 3.4401 \\
& Level 4 & $7.7543 \mathrm{e}-2$ & $2.5462 \mathrm{e}-3$ & 2.0763 & $9.2338 \mathrm{e}-5$ & 3.1651 \\
& Level 5 & $3.9258 \mathrm{e}-2$ & $6.3795 \mathrm{e}-4$ & 2.0334 & $1.1458 \mathrm{e}-5$ & 3.0657 \\
\hline 3 & Level 1 & $5.6522 \mathrm{e}-1$ & $2.0799 \mathrm{e}-2$ & & $9.2165 \mathrm{e}-3$ & \\
& Level 2 & $2.8553 \mathrm{e}-1$ & $2.3994 \mathrm{e}-3$ & 3.1627 & $3.9140 \mathrm{e}-4$ & 4.6261 \\
& Level 3 & $1.5110 \mathrm{e}-1$ & $2.9397 \mathrm{e}-4$ & 3.2990 & $2.0353 \mathrm{e}-5$ & 4.6456 \\
& Level 4 & $7.7543 \mathrm{e}-2$ & $3.6590 \mathrm{e}-5$ & 3.1235 & $1.1766 \mathrm{e}-6$ & 4.2731 \\
& Level 5 & $3.9258 \mathrm{e}-2$ & $4.5738 \mathrm{e}-6$ & 3.0549 & $7.3538 \mathrm{e}-8$ & 4.0733 \\
\hline
\end{tabular}

mesh generation and computation are all conducted in the MATLAB environment. Taking advantage of the availability of analytical solutions in each test example, the necessary boundary and interface conditions are all derived from the exact solutions.

Example 1. We first consider a circular interface problem. Here $\Omega_{1}$ is defined to be a circle with its center at the origin and its radius being 0.5 . Let $\Omega=(-1,1)^{2}$ and $\Omega_{2}=\Omega / \Omega_{1}$. The coefficient $A$ is chosen as $A_{1}=10$ and $A_{2}=1$, respectively, in $\Omega_{1}$ and $\Omega_{2}$. The analytical solution to the elliptic equation is given as

$$
u= \begin{cases}10-x^{2}-y^{2}, & \text { if }(x, y) \in \Omega_{1} \\ \sin (\pi x) \sin (\pi y), & \text { if }(x, y) \in \Omega_{2} .\end{cases}
$$

A sequence of uniform triangular meshes is generated in align with the interface $\Gamma$, i.e., $r=\sqrt{x^{2}+y^{2}}=0.5$. Based on these meshes, the $L^{2}$ errors of the piecewise linear WG-FEM for the solution and its gradient are reported in Table 4.1. The numerically detected convergence rates are also reported for successively refined meshes. Clearly, the orders of convergence are about two for the solution, and about one for the gradient. This agree with our theoretical estimates.

We next consider higher order WG finite element methods (WG-FEMs) with the polynomial degree being $k=2$ and $k=3$. Instead of computing the $L^{2}$ error, we check the edge-based $L^{2}$ error in the solution, which is defined as

$$
\left\|Q_{b} u-u_{b}\right\|=\left(\sum_{e \in \mathcal{E}_{h}} h \int_{e}\left(Q_{b} u-u_{b}\right)^{2} d s\right)^{1 / 2} .
$$




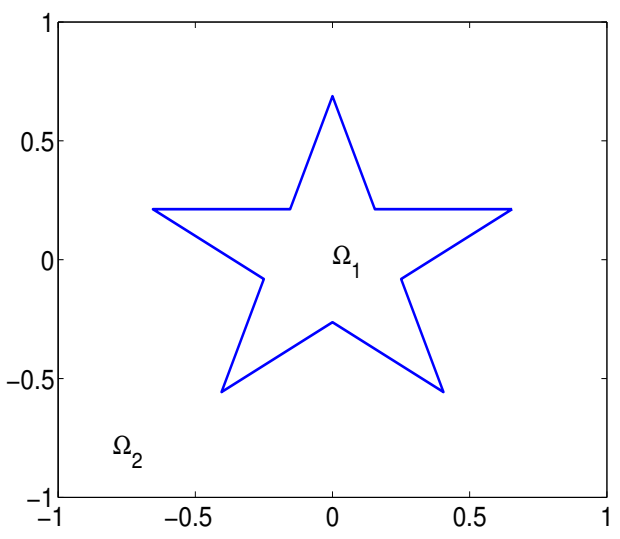

FIG. 4.1. The interface $\Gamma$ in Example 2.

TABLE 4.3

Numerical convergence of the piecewise linear WG-FEM for Example 2.

\begin{tabular}{|c|c|cc|cc|}
\hline Mesh & \multirow{2}{*}{$\max \{h\}$} & \multicolumn{2}{|c|}{ Gradient } & \multicolumn{2}{c|}{ Solution } \\
\cline { 3 - 6 } & & $L^{2}$ error & order & $L^{2}$ error & order \\
\hline Level 1 & $4.7778 \mathrm{e}-1$ & $1.8483 \mathrm{e}-0$ & & $5.6857 \mathrm{e}-1$ & \\
Level 2 & $2.3889 \mathrm{e}-1$ & $7.5111 \mathrm{e}-1$ & 1.2991 & $1.4087 \mathrm{e}-1$ & 2.0130 \\
Level 3 & $1.1944 \mathrm{e}-1$ & $3.4091 \mathrm{e}-1$ & 1.1396 & $3.5107 \mathrm{e}-2$ & 2.0044 \\
Level 4 & $5.9720 \mathrm{e}-2$ & $1.6283 \mathrm{e}-1$ & 1.0660 & $8.7630 \mathrm{e}-3$ & 2.0023 \\
Level 5 & $2.9860 \mathrm{e}-2$ & $7.9658 \mathrm{e}-2$ & 1.0315 & $2.1891 \mathrm{e}-3$ & 2.0011 \\
\hline
\end{tabular}

The numerical results are listed in Table 4.2. It can be seen that the numerical order in the gradient and solution is, respectively, $k$ and $k+1$. Thus, the optimal rates of convergence are achieved in these high order WG-FEMs.

Example 2. We next consider a five-point star interface embedded in the domain $\Omega=(0,1)^{2}$. The shape of the interface $\Gamma$ and two subdomains $\Omega_{1}$ and $\Omega_{2}$ are shown in Fig. 4.1. The coefficient $A$ is chosen as $A_{1}=10$ and $A_{2}=1$, respectively, in $\Omega_{1}$ and $\Omega_{2}$. The exact solution is taken as

$$
u(x, y)= \begin{cases}x-y^{2}+10, & \text { if }(x, y) \in \Omega_{1} \\ e^{x} \cos \pi y, & \text { if }(x, y) \in \Omega_{2} .\end{cases}
$$

The numerical errors of the piecewise linear WG-FEM and high order WG-FEMs are given in Table 4.3 and Table 4.4, respectively. Again, the optimal rates of convergence are achieved for all $k$ degrees. One numerical solution of the piecewise linear WG-FEM is shown in Fig. 4.2, which is indeed indistinguishable from the analytical solution.

Example 3. We further examine high order convergences of the WG method by considering a curved interface with both concave and convex segments. Here, $\Gamma$ is parametrized with the polar angle $\theta$

$$
\Gamma: \quad \sqrt{x^{2}+y^{2}}=\frac{1}{2}+\frac{1}{4} \sin (2 \theta), \quad \theta \in[0,2 \pi] .
$$

With the domain $\Omega=(0,1)^{2}, \Omega_{1}$ and $\Omega_{2}$ are set to be the subdomain inside and outside $\Gamma$, respectively. The coefficient $A$ is chosen to be $A_{1}=10$ and $A_{2}=1$ 
TABLE 4.4

Numerical convergence of high order WG-FEMs for Example 2.

\begin{tabular}{|c|cc|cc|cc|}
\hline $\mathrm{k}$ & Mesh & $\max \{h\}$ & \multicolumn{2}{|c|}{ Gradient } & \multicolumn{2}{c|}{ Solution } \\
\cline { 4 - 7 } & & & $L^{2}$ error & order & edge-based $L^{2}$ error & order \\
\hline 2 & Level 1 & $6.2411 \mathrm{e}-1$ & $1.1505 \mathrm{e}-1$ & & $3.9844 \mathrm{e}-2$ & \\
& Level 2 & $3.1205 \mathrm{e}-1$ & $3.2075 \mathrm{e}-2$ & 1.8427 & $4.8558 \mathrm{e}-3$ & 3.0365 \\
& Level 3 & $1.5603 \mathrm{e}-1$ & $8.3753 \mathrm{e}-3$ & 1.9373 & $5.6788 \mathrm{e}-4$ & 3.0962 \\
& Level 4 & $7.8013 \mathrm{e}-2$ & $2.1315 \mathrm{e}-3$ & 1.9742 & $6.7987 \mathrm{e}-5$ & 3.0621 \\
& Level 5 & $3.9007 \mathrm{e}-2$ & $5.3716 \mathrm{e}-4$ & 1.9885 & $8.3146 \mathrm{e}-6$ & 3.0316 \\
\hline 3 & Level 1 & $6.2411 \mathrm{e}-1$ & $1.2848 \mathrm{e}-2$ & & $5.3619 \mathrm{e}-3$ & \\
& Level 2 & $3.1205 \mathrm{e}-1$ & $1.7762 \mathrm{e}-3$ & 2.8546 & $3.0383 \mathrm{e}-4$ & 4.1413 \\
& Level 3 & $1.5603 \mathrm{e}-1$ & $2.2733 \mathrm{e}-4$ & 2.9661 & $1.6949 \mathrm{e}-5$ & 4.1642 \\
& Level 4 & $7.8013 \mathrm{e}-2$ & $2.9145 \mathrm{e}-5$ & 2.9634 & $9.8627 \mathrm{e}-7$ & 4.1029 \\
& Level 5 & $3.9007 \mathrm{e}-2$ & $3.6430 \mathrm{e}-6$ & 3.0001 & $6.1742 \mathrm{e}-8$ & 3.9977 \\
\hline
\end{tabular}
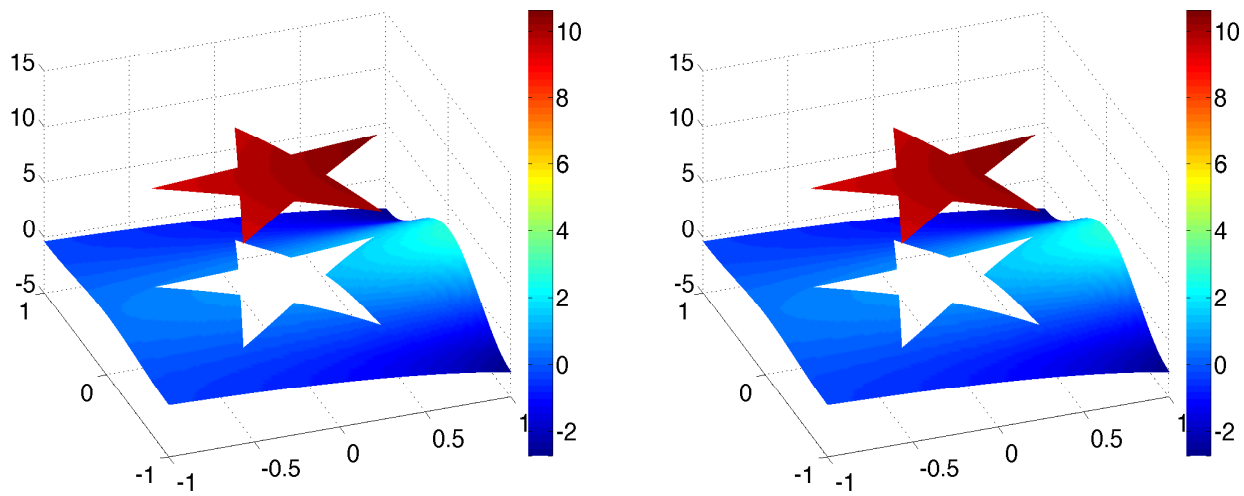

FIG. 4.2. The piecewise linear WG approximation of Example 2 on the mesh level 5. Left: Numerical solution; Right: Exact solution.

correspondingly. We also set the analytic solution as

$$
u(x, y)= \begin{cases}x-y^{2}+10, & \text { if }(x, y) \in \Omega_{1} \\ e^{x} \cos \pi y, & \text { if }(x, y) \in \Omega_{2} .\end{cases}
$$

We again report the numerical errors in two tables: Table 4.5 and Table 4.6, because the $L^{2}$ norms used in the solution tests are slightly different for the piecewise linear and high order WG-FEMs. It can be seen that for the present non-constant curvature interface, the desired high order convergences are also successfully achieved in the WG method. In Fig. 4.2, the piecewise linear WG solutions on mesh level 1 and 5 are depicted. Based on a coarse grid, the discontinuous approximation of the WG method over different finite elements can be clearly observed in the mesh level 1. But when a fine mesh is employed, the WG solution appears to be quite smooth for regions away from the interface $\Gamma$.

In Examples 1, 2, and 3, the high order convergences of the proposed WG method for solving elliptic interface problem are demonstrated. In the following examples, we will investigate the robustness of the WG method in treating low regularity solutions and complex geometry. Only the piecewise linear WG-FEM will be considered for 
TABLE 4.5

Numerical convergence of the piecewise linear WG-FEM for Example 3.

\begin{tabular}{|c|c|cc|cc|}
\hline Mesh & \multirow{2}{*}{$\max \{h\}$} & \multicolumn{2}{|c|}{ Gradient } & \multicolumn{2}{c|}{ Solution } \\
\cline { 3 - 6 } & & $L^{2}$ error & order & $L^{2}$ error & order \\
\hline Level 1 & $3.5804 \mathrm{e}-1$ & $9.2424 \mathrm{e}-1$ & & $2.6947 \mathrm{e}-1$ & \\
Level 2 & $1.7902 \mathrm{e}-1$ & $4.1898 \mathrm{e}-1$ & 1.1414 & $6.6910 \mathrm{e}-2$ & 2.0098 \\
Level 3 & $8.9509 \mathrm{e}-2$ & $2.0142 \mathrm{e}-1$ & 1.0567 & $1.6698 \mathrm{e}-2$ & 2.0025 \\
Level 4 & $4.4754 \mathrm{e}-2$ & $9.8997 \mathrm{e}-2$ & 1.0247 & $4.1723 \mathrm{e}-3$ & 2.0007 \\
Level 5 & $2.2377 \mathrm{e}-2$ & $4.9499 \mathrm{e}-2$ & 1.0000 & $1.0431 \mathrm{e}-3$ & 2.0000 \\
\hline
\end{tabular}

TABLE 4.6

Numerical convergence of high order WG-FEMs for Example 3.

\begin{tabular}{|c|cc|cc|cc|}
\hline \multirow{2}{*}{$\mathrm{k}$} & Mesh & \multirow{2}{*}{$\max \{h\}$} & \multicolumn{2}{|c|}{ Gradient } & \multicolumn{2}{c|}{ Solution } \\
\cline { 4 - 7 } & & & $L^{2}$ error & order & edge-based $L^{2}$ error & order \\
\hline 2 & Level 1 & $4.5260 \mathrm{e}-1$ & $1.4543 \mathrm{e}-1$ & & $5.1843 \mathrm{e}-2$ & \\
& Level 2 & $2.2630 \mathrm{e}-1$ & $4.0485 \mathrm{e}-2$ & 1.8448 & $6.0126 \mathrm{e}-3$ & 3.1080 \\
& Level 3 & $1.1315 \mathrm{e}-1$ & $1.0643 \mathrm{e}-2$ & 1.9275 & $7.3099 \mathrm{e}-4$ & 3.0401 \\
& Level 4 & $5.6575 \mathrm{e}-2$ & $2.7281 \mathrm{e}-3$ & 1.9639 & $9.1061 \mathrm{e}-5$ & 3.0049 \\
\hline 3 & Level 1 & $4.5260 \mathrm{e}-1$ & $1.3353 \mathrm{e}-2$ & & $4.5656 \mathrm{e}-3$ & \\
& Level 2 & $2.2630 \mathrm{e}-1$ & $1.9367 \mathrm{e}-3$ & 2.7854 & $3.2544 \mathrm{e}-4$ & 3.8103 \\
& Level 3 & $1.1315 \mathrm{e}-1$ & $2.5154 \mathrm{e}-4$ & 2.9447 & $1.8976 \mathrm{e}-5$ & 4.1001 \\
& Level 4 & $5.6575 \mathrm{e}-2$ & $3.2395 \mathrm{e}-5$ & 2.9569 & $1.1511 \mathrm{e}-6$ & 4.0431 \\
\hline
\end{tabular}
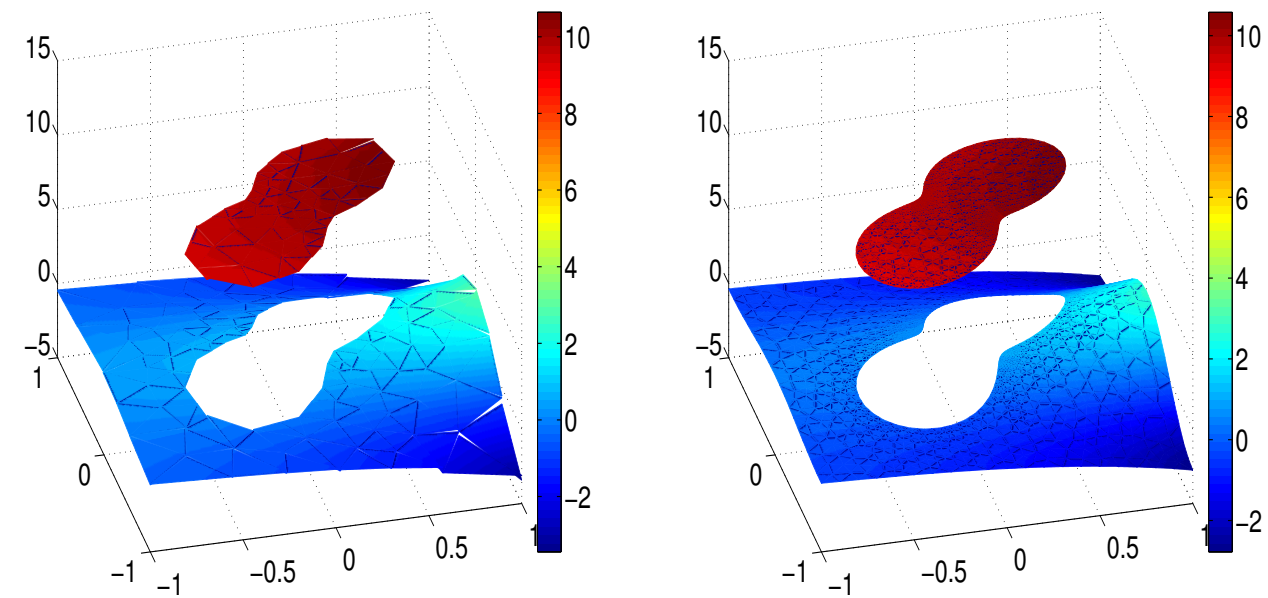

FIG. 4.3. The piecewise linear WG approximation of Example 3. Left: mesh level 1; Right: mesh level 5.

simplicity.

Example 4. In this and next two examples, we study three interface problems defined geometrically on the same interface and domain [26, 27, 37], while solutions of different regularities will be studied. Because it is customary to report only $L_{\infty}$ errors for solutions of low regularities, we will only present $L_{\infty}$ errors in these three examples as well. 
TABLE 4.7

Numerical convergence of the piecewise linear WG-FEM for Example 4.

\begin{tabular}{|c|c|cc|cc|}
\hline Mesh & $\max \{h\}$ & \multicolumn{2}{|c|}{ Gradient } & \multicolumn{2}{c|}{ Solution } \\
\cline { 3 - 6 } & & $L_{\infty}$ error & order & $L_{\infty}$ error & order \\
\hline Level 1 & $9.7065 \mathrm{e}-1$ & $2.8652 \mathrm{e}-1$ & & $1.1788 \mathrm{e}-1$ & \\
Level 2 & $4.8533 \mathrm{e}-1$ & $1.5217 \mathrm{e}-1$ & 0.9130 & $2.9692 \mathrm{e}-2$ & 1.9892 \\
Level 3 & $2.4266 \mathrm{e}-1$ & $7.6254 \mathrm{e}-2$ & 0.9968 & $7.7789 \mathrm{e}-3$ & 1.9324 \\
Level 4 & $1.2133 \mathrm{e}-1$ & $3.8168 \mathrm{e}-2$ & 0.9984 & $1.9575 \mathrm{e}-3$ & 1.9906 \\
Level 5 & $6.0666 \mathrm{e}-2$ & $1.9089 \mathrm{e}-2$ & 0.9997 & $4.9101 \mathrm{e}-4$ & 1.9952 \\
\hline
\end{tabular}

On a rectangular domain $\Omega=(-1,3) \times(-1,1)$, we consider a Lipschitz continuous interface $\Gamma$ that consists of two pieces: $y=2 x$ for $x+y>0$ and $y=-x / 2$ for $x+y \leq 0$. Thus, the interface $\Gamma$ has a kink at $(0,0)$. Denote by $\Omega_{1}$ the region on the left and upper part of $\Gamma$ and $\Omega_{2}$ to be the rest of the domain. The coefficient is given piecewisely as

$$
A(x, y)= \begin{cases}(x y+2) / 5, & \text { if }(x, y) \in \Omega_{1} \\ \left(x^{2}-y^{2}+3\right) / 7, & \text { if }(x, y) \in \Omega_{2}\end{cases}
$$

The analytical solution is chosen as

$$
u(x, y)= \begin{cases}8, & \text { if }(x, y) \in \Omega_{1} \\ \sin (x+y), & \text { if }(x, y) \in \Omega_{2} \text { and if } x+y \leq 0 \\ x+y, & \text { if }(x, y) \in \Omega_{2} \text { and if } x+y>0\end{cases}
$$

The $L_{\infty}$ errors of the piecewise linear WG-FEM are shown in Table 4.7. We note that the present analytical solution is designed to be of $C^{2}$ continuous but not $C^{3}$. For this solution, the proposed WG method attains second and first order of convergence, respectively, for the solution and its gradient. The numerical orders appear to be very uniform and are very close to the theoretical ones.

Example 5. Here we consider the same interface and domain geometry as in Example 4. The coefficient function $A(x, y)$ is defined in the same way as in Example 4. The analytical solution is now chosen as

$$
u(x, y)= \begin{cases}8, & \text { if }(x, y) \in \Omega_{1} \\ \sin (x+y)+\cos (x+y), & \text { if }(x, y) \in \Omega_{2} \text { and if } x+y \leq 0 \\ x+y+1, & \text { if }(x, y) \in \Omega_{2} \text { and if } x+y>0\end{cases}
$$

We note that the analytical solution is $C^{1}$ continuous but not $C^{2}$, across the line $x+y=0$ in $\Omega_{2}$. But in the present computation, no special boundary or interface treatment is adopted near this line, so that this line actually cuts through the finite element triangles. Numerically, since the regularity of the solution is low, a reduction in convergence rate could happen in computation. The previous WG method [37] has been found to be very robust in handling such kind of solutions, without order reduction. The $L_{\infty}$ errors of the present WG-FEM are shown in Table 4.8. It is can be seen that the numerical convergence rates of the piecewise linear WG-FEM are not very uniform. But the new WG-FEM eventually delivers a second and first order of convergence for the solution and its gradient, respectively. 
TABLE 4.8

Numerical convergence of the piecewise linear WG-FEM norm for Example 5.

\begin{tabular}{|c|c|cc|cc|}
\hline Mesh & \multirow{2}{*}{$\max \{h\}$} & \multicolumn{2}{|c|}{ Gradient } & \multicolumn{2}{c|}{ Solution } \\
\cline { 3 - 6 } & & $L_{\infty}$ error & order & $L_{\infty}$ error & order \\
\hline Level 1 & $9.7065 \mathrm{e}-1$ & $1.8919 \mathrm{e}-1$ & & $1.1146 \mathrm{e}-1$ & \\
Level 2 & $4.8533 \mathrm{e}-1$ & $1.5991 \mathrm{e}-1$ & 0.2426 & $3.6753 \mathrm{e}-2$ & 1.6006 \\
Level 3 & $2.4266 \mathrm{e}-1$ & $9.2269 \mathrm{e}-2$ & 0.7933 & $1.0049 \mathrm{e}-2$ & 1.8708 \\
Level 4 & $1.2133 \mathrm{e}-1$ & $4.8593 \mathrm{e}-2$ & 0.9251 & $2.5968 \mathrm{e}-3$ & 1.9522 \\
Level 5 & $6.0666 \mathrm{e}-2$ & $2.4828 \mathrm{e}-2$ & 0.9688 & $6.6317 \mathrm{e}-4$ & 1.9693 \\
\hline
\end{tabular}

TABLE 4.9

Numerical convergence of the piecewise linear WG-FEM for Example 6.

\begin{tabular}{|c|c|cc|cc|}
\hline Mesh & \multirow{2}{*}{$\max \{h\}$} & \multicolumn{2}{|c|}{ Gradient } & \multicolumn{2}{c|}{ Solution } \\
\cline { 3 - 6 } & & $L_{\infty}$ error & order & $L_{\infty}$ error & order \\
\hline Level 1 & $9.7065 \mathrm{e}-1$ & $3.5133 \mathrm{e}-1$ & & $5.6605 \mathrm{e}-1$ & \\
Level 2 & $4.8533 \mathrm{e}-1$ & $1.9424 \mathrm{e}-1$ & 0.8550 & $1.7517 \mathrm{e}-1$ & 1.6922 \\
Level 3 & $2.4266 \mathrm{e}-1$ & $9.9309 \mathrm{e}-2$ & 0.9678 & $4.7433 \mathrm{e}-2$ & 1.8847 \\
Level 4 & $1.2133 \mathrm{e}-1$ & $5.0195 \mathrm{e}-2$ & 0.9844 & $1.2260 \mathrm{e}-2$ & 1.9519 \\
Level 5 & $6.0666 \mathrm{e}-2$ & $2.5606 \mathrm{e}-2$ & 0.9801 & $3.1116 \mathrm{e}-3$ & 1.9783 \\
\hline
\end{tabular}

Example 6. Consider the same interface and geometry as in Examples 4 and 5. The coefficient function is now defined to be

$$
A(x, y)= \begin{cases}1, & \text { if }(x, y) \in \Omega_{1}, \\ 2+\sin (x+y), & \text { if }(x, y) \in \Omega_{2} .\end{cases}
$$

The analytical solution is set to be

$$
u(x, y)= \begin{cases}8, & \text { if }(x, y) \in \Omega_{1}, \\ \left(x^{2}+y^{2}\right)^{5 / 6}+\sin (x+y), & \text { if }(x, y) \in \Omega_{2} .\end{cases}
$$

We note that the present analytical solution is piecewise $H^{2}$. The solution $u(x, y)$ has a singularity at $(0,0)$ with blow-up derivatives, and the source $f$ goes to infinity at the origin. In the WG computations, no special treatment is needed for a singular source located on a vertex of triangles. A 12-points Gaussian quadrature is used in all elements for the numerical integration of $\left(f, v_{0}\right)$, where $v_{0}$ is defined within the interior of each element. In solving this challenging problem with low regularity, our previous WG method only attains a order of 1.75 in the $L_{\infty}$ norm for the solution [37]. However, the new WG-FEM yields a fully second order of accuracy in the $L_{\infty}$ norm, see Table 4.9. In other words, the proposed WG-FEM still achieves the optimal rates of convergence for both solution and gradient for this low regularity example. The improvement of the present WG algorithm over the previous WG algorithm is believed to be due to the use of piecewise linear basis functions in the present studies. The previous WG studies are based on piecewise constant basis functions [37].

Example 7. After examining the robustness of the proposed WG method in dealing with solutions of low regularities, we next study the capability of the WG method for accommodating complex geometries. We first showcase an example by illustrating how the new WG method admits general finite element partitions con- 

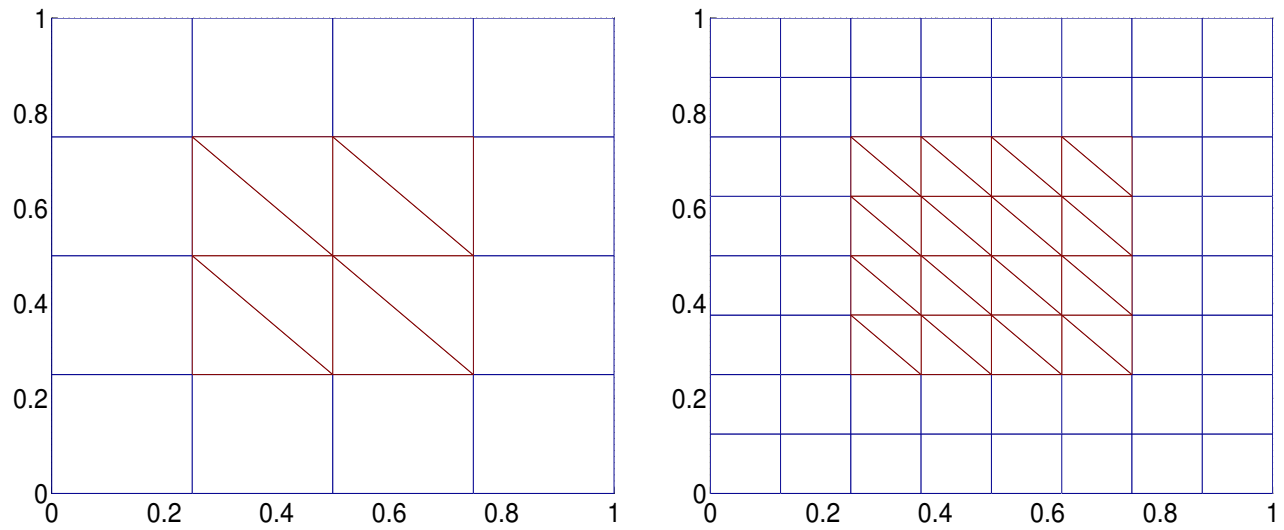

FIG. 4.4. The mixed meshes of Example 7. Left: mesh level 1; Right: mesh level 2.

sisting of arbitrary polytopal elements. For simplicity, we only consider the piecewise linear WG-FEM $P_{1}(K)-P_{1}(e)$, and errors are reported in the $L^{2}$ norm.

A simple square interface is considered. Let the domain $\Omega=(0,1)^{2}$ with $\Omega_{1}=$ $[0.2,0.8]^{2}$ and $\Omega_{2}=\Omega / \Omega_{2}$. The coefficient function is taken as

$$
A(x, y)= \begin{cases}1, & \text { if }(x, y) \in \Omega_{1} \\ 2+\sin (x+y), & \text { if }(x, y) \in \Omega_{2}\end{cases}
$$

Then the exact solution is chosen as follows:

$$
u= \begin{cases}5+5\left(x^{2}+y^{2}\right), & \text { if }(x, y) \in \Omega_{1} \\ x^{2}+y^{2}+\sin (x+y), & \text { if }(x, y) \in \Omega_{2}\end{cases}
$$

Different types of meshes are employed in the present WG algorithm. In particular, in $\Omega_{1}$, a uniform triangular mesh is used, while in $\Omega_{2}$, a uniform rectangular mesh is generated. The initial mixed mesh is shown in the left chart of Fig.4.4. Then by connecting the middle points on the edges, the refinement of previous level of mesh is derived. The next level mixed mesh is given in the right chart of Fig. 4.4.

The numerical errors of the piecewise linear WG method are reported in Table 4.10. Obviously, the theoretical orders, i.e., $O\left(h^{2}\right)$ in $L^{2}$ norm and $O(h)$ in $H^{1}$ norm, are achieved based on such polytopal elements. The WG solutions on the coarsest and finest meshes are depicted in Fig. 4.5. Because of the high accuracy of the WG algorithm and interface being perfectly represented by the mixed meshes, one could not see much differences between these two WG solutions.

Example 8. We next consider an arbitrarily shaped interface $\Gamma$, whose parametric form is given as

$$
\left\{\begin{array}{l}
x(\theta)=(a+b \cos (m \theta) \sin (n \theta)) \cos (\theta), \\
y(\theta)=(a+b \cos (m \theta) \sin (n \theta)) \sin (\theta),
\end{array}\right.
$$

for $\theta \in[0,2 \pi]$. Here we choose $a=b=0.40178, m=2$, and $n=6$. Over the domain $\Omega=(-1,1)^{2}, \Omega_{1}$ and $\Omega_{2}$ is defined, respectively, to be the region inside and outside $\Gamma$. The interface and subdomains are illustrated in the left chart of Fig. 4.6. In our 
TABLE 4.10

Numerical convergence of the piecewise linear WG-FEM for Example $\%$.

\begin{tabular}{|c|c|cc|cc|}
\hline \multirow{2}{*}{ Mesh } & \multirow{2}{*}{$h$} & \multicolumn{2}{|c|}{ Gradient } & \multicolumn{2}{c|}{ Solution } \\
\cline { 3 - 6 } & & $L^{2}$ error & order & $L^{2}$ error & order \\
\hline Level 1 & $1 / 4$ & $2.1406 \mathrm{e}-1$ & & $1.3885 \mathrm{e}-1$ & \\
Level 2 & $1 / 8$ & $8.7942 \mathrm{e}-2$ & 1.2834 & $3.4667 \mathrm{e}-2$ & 2.0019 \\
Level 3 & $1 / 16$ & $3.9064 \mathrm{e}-2$ & 1.1707 & $8.6661 \mathrm{e}-3$ & 2.0001 \\
Level 4 & $1 / 64$ & $8.8181 \mathrm{e}-3$ & 1.0511 & $5.4165 \mathrm{e}-4$ & 2.0000 \\
Level 5 & $1 / 128$ & $4.3293 \mathrm{e}-3$ & 1.0263 & $1.3541 \mathrm{e}-4$ & 2.0000 \\
\hline
\end{tabular}
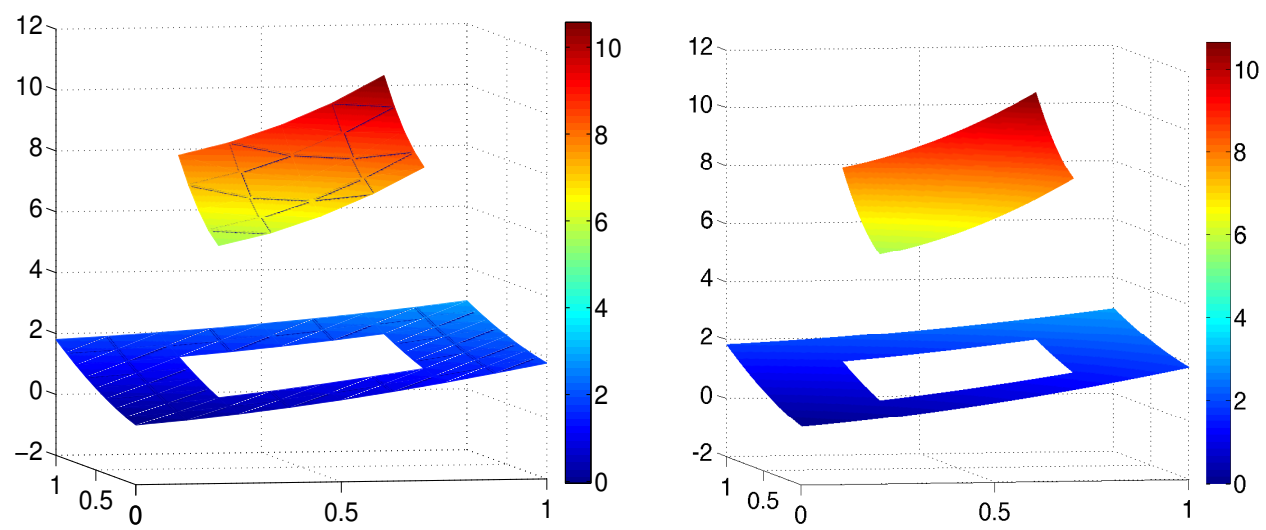

FIG. 4.5. The WG approximation of Example 7. Left: Mesh level 1; Right: mesh level 5.

test, the coefficient function is taken as

$$
A(x, y)= \begin{cases}\left(x^{2}-y^{2}+3\right) / 7, & \text { if }(x, y) \in \Omega_{1}, \\ (x y+2) / 5, & \text { if }(x, y) \in \Omega_{2} .\end{cases}
$$

The analytical solution is set as

$$
u= \begin{cases}\sin (x+y)+\cos (x+y)+1, & \text { if }(x, y) \in \Omega_{1}, \\ x+y+1, & \text { if }(x, y) \in \Omega_{2} .\end{cases}
$$

The $L^{2}$ errors of the piecewise linear WG-FEM are listed in Table 4.11. With successive mesh refinements, the WG method is able to deliver second order of accuracy in solution for this challenging problem. The WG solutions are shown in Fig. 4.7. It can be seen that the approximation of $\Gamma$ by level 1 mesh is very crude. Nevertheless, the WG solution has already captured the main features of the solution at mesh level 1. At mesh level 4, the numerical solution is visually the same as the analytical solution.

Example 9. In this example, the same parametric equations of interface as in Example 8 are considered. Here, we take $a=0.50012563, b=0.250012563, m=0$, and $n=12$. The same problem setting and analytical solution as in Example 8 are also employed. The shape of $\Gamma$ is shown in the middle chart of Fig. 4.6. The numerical results are given in Table 4.12. Again, the WG method attains the optimal rate of convergence. The numerical solutions are shown in Fig. 4.8. 

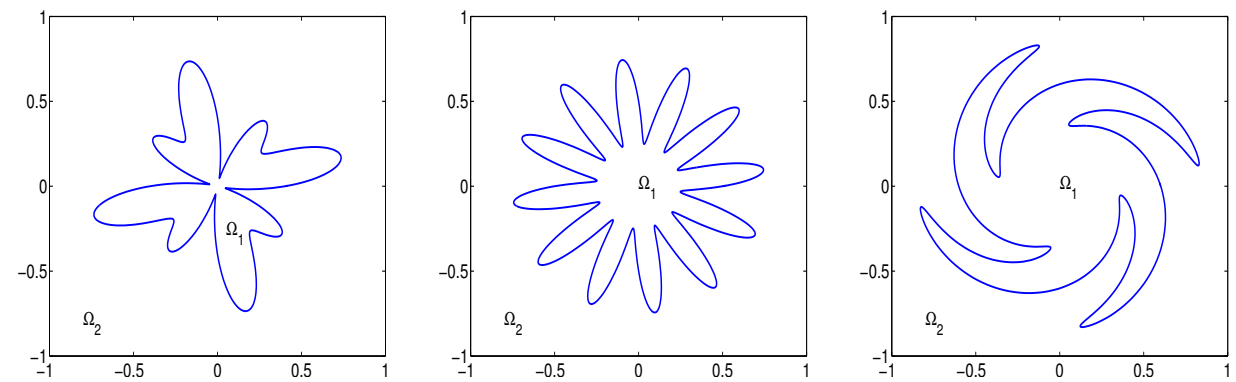

Fig. 4.6. The plot of interface $\Gamma$ in Example 8 (Left), Example 9 (Middle), and Example 10 (Right). The inside region is denoted as $\Omega_{1}$, while the outside one is denoted as $\Omega_{2}$.

TABLE 4.11

Numerical convergence of the piecewise linear WG-FEM for Example 8.

\begin{tabular}{|c|c|cc|cc|}
\hline Mesh & \multirow{2}{*}{$\max \{h\}$} & \multicolumn{2}{|c|}{ Gradient } & \multicolumn{2}{c|}{ Solution } \\
\cline { 3 - 6 } & & $L^{2}$ error & order & $L^{2}$ error & order \\
\hline Level 1 & $2.0697 \mathrm{e}-1$ & $4.9000 \mathrm{e}-2$ & & $1.0491 \mathrm{e}-2$ & \\
Level 2 & $1.0348 \mathrm{e}-1$ & $2.4739 \mathrm{e}-2$ & 0.9860 & $2.6227 \mathrm{e}-3$ & 2.0000 \\
Level 3 & $5.1740 \mathrm{e}-2$ & $1.1992 \mathrm{e}-2$ & 1.0447 & $6.5239 \mathrm{e}-4$ & 2.0072 \\
Level 4 & $2.5870 \mathrm{e}-2$ & $5.8415 \mathrm{e}-3$ & 1.0377 & $1.6255 \mathrm{e}-4$ & 2.0049 \\
\hline
\end{tabular}
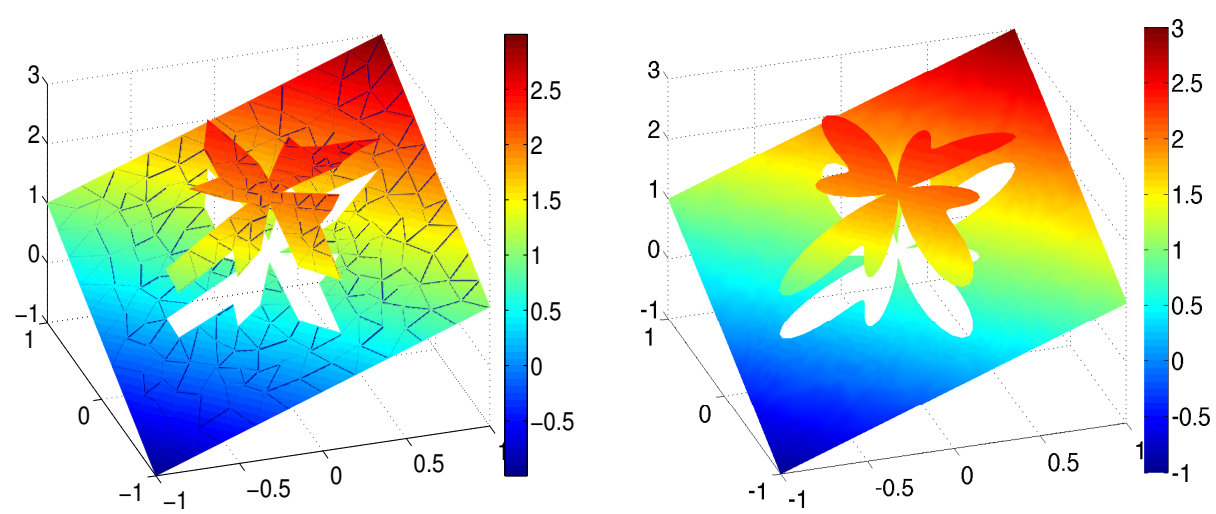

FIG. 4.7. The WG approximation of Example 8 on mesh: level 1 (Left) and level 4 (Right).

TABLE 4.12

Numerical convergence of the piecewise linear WG-FEM for Example 9.

\begin{tabular}{|c|c|cc|cc|}
\hline Mesh & \multirow{2}{*}{$\max \{h\}$} & \multicolumn{2}{|c|}{ Gradient } & \multicolumn{2}{c|}{ Solution } \\
\cline { 3 - 6 } & & $L^{2}$ error & order & $L^{2}$ error & order \\
\hline Level 1 & $2.6956 \mathrm{e}-1$ & $2.7192 \mathrm{e}-2$ & & $3.0386 \mathrm{e}-3$ & \\
Level 2 & $1.3478 \mathrm{e}-1$ & $1.3081 \mathrm{e}-2$ & 1.0557 & $7.5792 \mathrm{e}-4$ & 2.0033 \\
Level 3 & $6.7391 \mathrm{e}-2$ & $6.3372 \mathrm{e}-3$ & 1.0456 & $1.8887 \mathrm{e}-4$ & 2.0047 \\
Level 4 & $3.3696 \mathrm{e}-2$ & $3.1686 \mathrm{e}-3$ & 1.0000 & $4.7217 \mathrm{e}-5$ & 2.0000 \\
\hline
\end{tabular}

Example 10. We finally consider a complex interface problem with high-contrast 

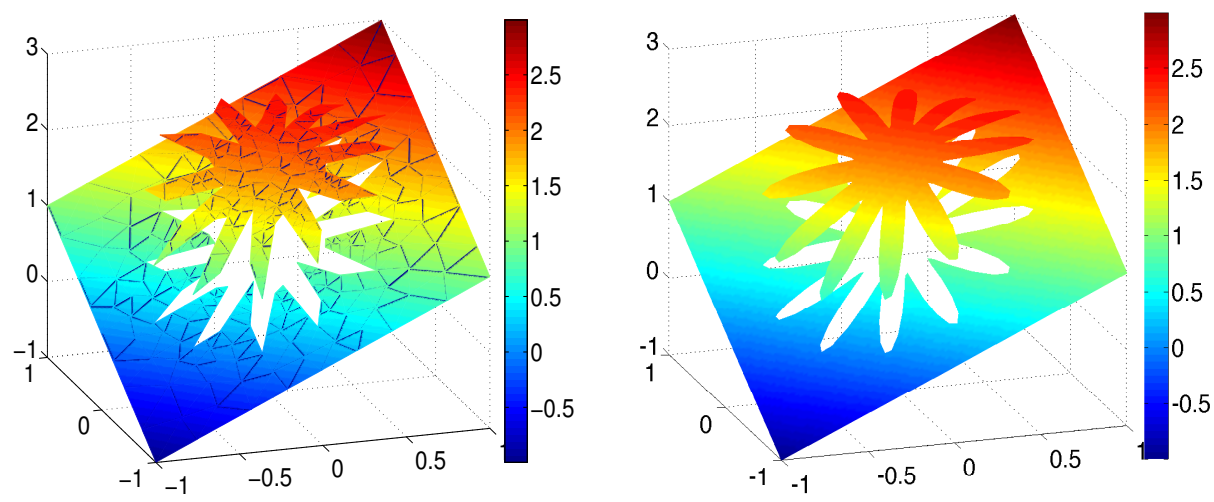

FIG. 4.8. The WG approximation of Example 9 on mesh: level 1 (Left) and level 4 (Right).

coefficients. The parametric equations of $\Gamma$ are given as

$$
\left\{\begin{array}{l}
x(t)=r(t) \cos (\theta(t)), \\
y(t)=r(t) \sin (\theta(t))
\end{array}\right.
$$

where

$$
\theta(t)=t+\sin (4 t), r(t)=0.6012563+0.2401256 \cos (4 t+\pi / 2) .
$$

Over the domain $\Omega=(-1,1)^{2}, \Omega_{1}$ and $\Omega_{2}$ is defined, respectively, to be the region inside and outside $\Gamma$. The interface and subdomains are illustrated in the right chart of Fig. 4.6. The exact solution is chosen as

$$
u(x, y)= \begin{cases}e^{x} \cos (y), & \text { if }(x, y) \in \Omega_{1}, \\ 0, & \text { if }(x, y) \in \Omega_{2},\end{cases}
$$

and the coefficient is set to be

$$
A(x, y)= \begin{cases}1+x^{2}+y^{2}, & \text { if }(x, y) \in \Omega_{1}, \\ b, & \text { if }(x, y) \in \Omega_{2} .\end{cases}
$$

In our test, we consider two high-contrast cases with $b=1000$ and $b=0.001$. The $L^{2}$ errors for both cases are shown in Table 4.13. It can be observed that the WG method is very robust in dealing with high-contrast coefficients. The same order of accuracies can be achieved in both the solution and its gradient for different $b$ values. The WG solutions for $b=1000$ are shown in Fig. 4.9.

5. Conclusion. This paper introduces a new weak Galerkin (WG) method for solving two-dimensional (2D) elliptic interface problems, which not only maintains various nice features of the previous WG algorithm [37], but also becomes more flexible and robust in handling complex interfaces. The WG method is a finite element method (FEM), in which differential operators are approximated by their weak forms as distributions. The first WG-FEM was introduced by Wang and Ye [45] and has been successfully applied to solve elliptic interface problems in [37]. Being flexible in 
TABLE 4.13

Numerical convergence of the piecewise linear WG-FEM for Example 10.

\begin{tabular}{|c|c|c|cc|cc|}
\hline$b$ & Mesh & $\max \{h\}$ & \multicolumn{2}{|c|}{ Gradient } & \multicolumn{2}{c|}{ Solution } \\
\cline { 4 - 7 } & & & $L^{2}$ error & order & $L^{2}$ error & order \\
\hline 1000 & Level 1 & $1.9077 \mathrm{e}-1$ & $4.0176 \mathrm{e}-2$ & & $1.7903 \mathrm{e}-3$ & \\
& Level 2 & $9.5386 \mathrm{e}-2$ & $1.9329 \mathrm{e}-2$ & 1.0556 & $4.4891 \mathrm{e}-4$ & 1.9957 \\
& Level 3 & $4.7693 \mathrm{e}-2$ & $9.3263 \mathrm{e}-3$ & 1.0514 & $1.1144 \mathrm{e}-4$ & 2.0102 \\
& Level 4 & $2.3846 \mathrm{e}-2$ & $4.6631 \mathrm{e}-3$ & 1.0000 & $2.7860 \mathrm{e}-5$ & 1.9999 \\
\hline 0.001 & Level 1 & $1.9077 \mathrm{e}-1$ & $8.4334 \mathrm{e}-2$ & & $3.7187 \mathrm{e}-3$ & \\
& Level 2 & $9.5386 \mathrm{e}-2$ & $2.7736 \mathrm{e}-2$ & 1.6044 & $7.2917 \mathrm{e}-4$ & 2.3505 \\
& Level 3 & $4.7693 \mathrm{e}-2$ & $1.1139 \mathrm{e}-2$ & 1.3161 & $1.5947 \mathrm{e}-4$ & 2.1930 \\
& Level 4 & $2.3846 \mathrm{e}-2$ & $5.5695 \mathrm{e}-3$ & 1.0000 & $3.9867 \mathrm{e}-5$ & 2.0000 \\
\hline
\end{tabular}
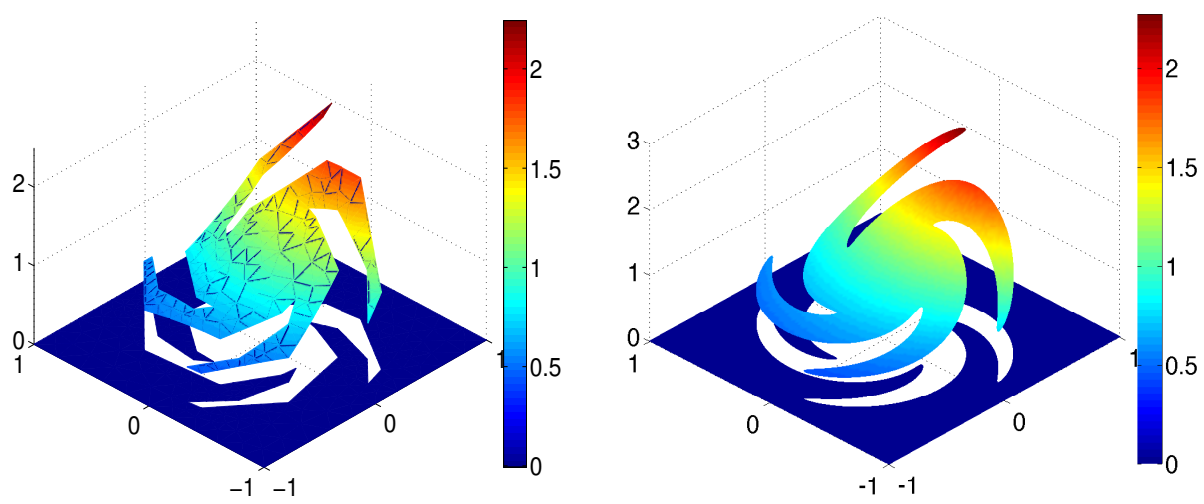

FIG. 4.9. The WG approximation of Example $10(b=1000)$ on mesh: level 1 (Left) and level 4 (Right).

enforcing boundary and interface conditions in the WG formulation, the WG interface approach can produce high order convergences in treating challenging elliptical solutions with low regularities. Recently, improved WG-FEMs have been constructed in $[46,38]$ which make use of a parameter-free stabilization in the WG formulation and allow the use of general partitions consisting of arbitrary polytopal elements. In the present study, we propose a new WG interface approach based on the WG-FEMs in $[46,38]$ and analyze its convergence rigorously. Such a WG formulation turns out to be much simpler than the previous one [37] for elliptic interface problems. Extensive numerical studies have been conducted to validate the proposed WG method in solving elliptic interface problems. In dealing with problems with low solution regularities, the new WG method outperforms the the previous WG method by delivering a uniform second order for all tested problems. Such an improvement is believed to be due to the use of piecewise linear basis functions in the present WG formulation, while the previous WG results were produced by using piecewise constant basis functions [37]. Moreover, the new WG method is easier to be generalized to higher order. We have demonstrate such high order of convergences by considering different interface problems. The numerical convergence rates agree with our theoretical estimates very well. Furthermore, we have studied several problems with very complex interface geometries, which can not be handled by the previous WG method, while the flexibil- 
ity of the new WG method in choosing finite element partitions enables an accurate approximation to these difficult problems.

\section{REFERENCES}

[1] I. Babuška. The finite element method for elliptic equations with discontinuous coefficients. Computing, 5:207-213, 1970.

[2] J. Bedrossian, J. H. von Brecht, S. W. Zhu, E. Sifakis, and J. M. Teran. A finite element method for interface problems in domains with smooth boundaries and interfaces. $J$. Comput. Phys., 229:6405-6426, 2010.

[3] P. A. Berthelsen. A decomposed immersed interface method for variable coefficient elliptic equations with non-smooth and discontinuous solutions. J. Comput. Phys., 197:364-386, 2004.

[4] J. Bramble and J. King. A finite element method for interface problems in domains with smooth boundaries and interfaces. Adv. Comput. Math., 6:109-138, 1996.

[5] F. Brezzi, K. Lipnikov, and M. Shashkov. Two families of mixed finite elements for second order elliptic problems. Numer. Math., 47:277-235, 1985.

[6] E. Burman and P. Hansbo. Interior-penalty-stabilized Lagrange multiplier methods for the finite-element solution of elliptic interface problems. IAM J. Numer. Anal., 30:870-885, 2010.

[7] Z. Cai, X. Ye, and S. Zhang. Discontinuous Galerkin finite element methods for interface problems: A priori and a posteriori error estimations. SIAM J. Numer. Anal., 49:17611787, 2011.

[8] D. Chen, Z. Chen, C. Chen, W. H. Geng, and G. W. Wei. MIBPB: A software package for electrostatic analysis. J. Comput. Chem., 32:657 - 670, 2011.

[9] T. Chen and J. Strain. Piecewise-polynomial discretization and Krylov-accelerated multigrid for elliptic interface problems. J. Comput. Phys., 16:7503-7542, 2008.

[10] Z. Chen and J. Zou. Finite element methods and their convergence for elliptic and parabolic interface problems. Numer. Math., 79:175-2002, 1998.

[11] I.-L. Chern and Y.-C. Shu. A coupling interface method for elliptic interface problems. J. Comput. Phys., 225:2138-2174, 2007.

[12] M. Dryjaa, J. Galvisb, and M. Sarkisb. BDDC methods for discontinuous Galerkin discretization of elliptic problems. Journal of Complexity, 23:715-739, 2007.

[13] R. E. Ewing, Z. L. Li, T. Lin, and Y. P. Lin. The immersed finite volume element methods for the elliptic interface problems. Mathematics and Computers in Simulation, 50:63-76, 1999.

[14] R. P. Fedkiw, T. Aslam, B. Merriman, and S. Osher. A non-oscillatory Eulerian approach to interfaces in multimaterial flows (the ghost fluid method). J. Comput. Phys., 152:457-492, 1999.

[15] W. H. Geng, S. N. Yu, and G. W. Wei. Treatment of charge singularities in implicit solvent models. Journal of Chemical Physics, 127:114106, 2007.

[16] R. Glowinski, T.-W. Pan, and J. Periaux. A fictitious domain method for Dirichlet problem and applications. Computer Methods in Applied Mechanics and Engineering, 111:283-303, 1994.

[17] G. Guyomarc'h, C.O. Lee, and K. Jeon. A discontinuous Galerkin method for elliptic interface problems with application to electroporation. Communications in Numerical Methods in Engineering, 25:991-1008, 2009.

[18] G. R. Hadley. High-accuracy finite-difference equations for dielectric waveguide analysis i: uniform regions and dielectric interfaces. Journal of Lightwave Technology, 20:1210-1218, 2002.

[19] A. Hansbo and P. Hansbo. An unfitted finite element method. Comput. Methods Appl. Mech. Engng, 191:5537-5552, 2002.

[20] I. Harari and J. Dolbow. Analysis of an efficient finite element method for embedded interface problems. Comput. Math., 46:205-211, 2010.

[21] X. He, T. Lin, and Y. Lin. Interior penalty bilinear IFE discontinuous Galerkin methods for elliptic equations with discontinuous coefficient. Journal of System Science and Complexity, 23:467-483, 2010.

[22] J. L. Hellrung Jr., L. M. Wang, E. Sifakis, and J. M. Teran. A second order virtual node method for elliptic problems with interfaces and irregular domains in three dimensions. Journal of Computational Physics, 231:2015-2048, 2012.

[23] J. S. Hesthaven. High-order accurate methods in time-domain computational electromagnetics. 
a review. Advances in Imaging and Electron Physics, 127:59-123, 2003.

[24] R. Hiptmair, J. Li, and J. Zou. Convergence analysis of finite element methods for H(div;Omega)-elliptic interface problems. J. Numer. Math., 18:187-218, 2010.

[25] T. P. Horikis and W. L. Kath. Modal analysis of circular bragg fibers with arbitrary index profiles. Optics Lett., 31:3417-3419, 2006.

[26] S. M. Hou and X.-D. Liu. A numerical method for solving variable coefficient elliptic equation with interfaces. J. Comput. Phys., 202:411-445, 2005.

[27] S. M. Hou, W. Wang, and L. Q. Wang. Numerical method for solving matrix coefficient elliptic equation with sharp-edged interfaces. J. Comput. Phys., 229:7162-7179, 2010.

[28] S. M. Hou, P. Song, L. Q. Wang, and H. K. Zhao. A weak formulation for solving elliptic interface problems without body fitted grid. J. Comput. Phys., 249:80-959, 2013.

[29] T. Y. Hou, Z. L. Li, S. Osher, and H. K. Zhao. A hybrid method for moving interface problems with application to the hele-shaw flow. J. Comput. Phys., 134(2):236-252, 1997.

[30] L. N. T. Huynh, N. C. Nguyen, J. Peraire, and B. C. Khoo. A high-order hybridizable discontinuous Galerkin method for elliptic interface problems. International Journal for Numerical Methods in Engineering, 93:183-200, 2013.

[31] A. T. Layton. Using integral equations and the immersed interface method to solve immersed boundary problems with stiff forces. Comput. Fluids., 38:266-272, 2009.

[32] R. J. LeVeque and Z. L. Li. The immersed interface method for elliptic equations with discontinuous coefficients and singular sources. SIAM J. Numer. Anal., 31:1019-1044, 1994.

[33] Z. L. Li and K. Ito. Maximum principle preserving schemes for interface problems with discontinuous coefficients. SIAM J. Sci. Comput., 23:339-361, 2001.

[34] Z. L. Li and K. Ito. The immersed interface method - numerical solutions of PDEs involving interfaces and irregular domains, SIAM Forntiers in Applied Mathematics, 2006.

[35] R. Massjung. An unfitted discontinuous Galerkin method applied to elliptic interface problems. SIAM J. Numer. Anal., 50:33134-3162, 2012.

[36] A. Mayo. The fast solution of Poisson's and the biharmonic equations on irregular regions. SIAM J. Numer. Anal., 21:285-299, 1984.

[37] L. Mu, J. Wang, G.W. Wei, X. Ye, and S. Zhao. Weak Galerkin methods for second order elliptic interface problems. J. Comput. Phys., 250:106-125, 2013.

[38] L. Mu, J. Wang, and X. Ye. Weak Galerkin finite element methods on polytopal meshes. Int. J. Numer. Anal. Model., 12:31-53, 2015.

[39] M. Oevermann and R. Klein. A Cartesian grid finite volume method for elliptic equations with variable coefficients and embedded interfaces. J. Comput. Phys., 219:749-769, 2006.

[40] C. S. Peskin and D. M. McQueen. A 3-dimensional computational method for blood-flow in the heart. 1. immersed elastic fibers in a viscous incompressible fluid. J. Comput. Phys., 81:372-405, 1989.

[41] C. S. Peskin. Numerical analysis of blood flow in the heart. J. Comput. Phys., 25(3):220-252, 1977.

[42] I. Ramiere. Convergence analysis of the $q_{1}$-finite element method for elliptic problems with non-boundary-fitted meshes. Int. J. Numer. Meth. Engng, 75:1007-1052, 2008.

[43] P. A. Raviart and J. M. Thomas. A mixed finite element method for 2-nd order elliptic problems, volume 606 of Mathematical Aspects of Finite Element Methods. Lecture Notes in Mathematics Series, A. Dold and B. Eckmann (Eds.), pages 292-315. Springer:Berlin, 1977.

[44] D. A. Wang, R. Li, and N. N. Yan. An edge-based anisotropic mesh refinement algorithm and its application to interface problems. Commun. Comput. Phys., 8:511-540, 2010.

[45] J. Wang and X. Ye. A weak Galerkin finite element method for second-order elliptic problems. J. Comp. Appl. Math., 241:103-115, 2013.

[46] J. Wang and X. Ye. A weak Galerkin mixed finite element method for second order elliptic problems. Math. Comput., 83:2101-2126, 2014.

[47] X. S. Wang, L. T. Zhang, and L. W. Kam. On computational issues of immersed finite element methods. Journal of Computational Physics, 228:2535-2551, 2009.

[48] A. Weigmann and K. Bube. The explicit-jump immersed interface method: finite difference methods for PDEs with piecewise smooth solutions. SIAM J. Numer. Anal., 37:3827-862, 2000.

[49] K. N. Xia, M. Zhan, and G.-W. Wei. MIB Galerkin method for elliptic interface problems. Journal of Computational and Applied Mathematics, 272:195-220, 2014.

[50] K. N. Xia and G.-W. Wei. A Galerkin formulation of the MIB method for three dimensional elliptic interface problems. Computers and Mathematics with Applications, 68:719-745, 2014.

[51] W. J. Ying and W. C. Wang. A kernel-free boundary integral method for implicitly defined 
surfaces. J. Comput. Phys., 252:606-624, 2013.

[52] S. N. Yu and G. W. Wei. Three-dimensional matched interface and boundary (MIB) method for treating geometric singularities. J. Comput. Phys., 227:602-632, 2007.

[53] S. N. Yu, Y. Zhou, and G. W. Wei. Matched interface and boundary (MIB) method for elliptic problems with sharp-edged interfaces. J. Comput. Phys., 224(2):729-756, 2007.

[54] S. N. Yu, W. H. Geng, and G. W. Wei. Treatment of geometric singularities in implicit solvent models. Journal of Chemical Physics, 126:244108, 2007.

[55] S. Zhao. High order matched interface and boundary methods for the helmholtz equation in media with arbitrarily curved interfaces. J. Comput. Phys., 229:3155-3170, 2010.

[56] S. Zhao and G. W. Wei. High-order FDTD methods via derivative matching for Maxwell's equations with material interfaces. J. Comput. Phys., 200(1):60-103, 2004.

[57] Y. C. Zhou, S. Zhao, M. Feig, and G. W. Wei. High order matched interface and boundary method for elliptic equations with discontinuous coefficients and singular sources. $J$. Comput. Phys., 213(1):1-30, 2006. 